\title{
POSITIVE HARMONIC FUNCTIONS ON COMB-LIKE DOMAINS
}

\author{
Joanna Pres \\ University College Dublin, School of Mathematical Sciences \\ Belfield, Dublin 4, Ireland; joanna.t.pres@gmail.com, joanna.pres@ucd.ie
}

\begin{abstract}
This paper investigates positive harmonic functions on a domain which contains an infinite cylinder, and whose boundary is contained in the union of parallel hyperplanes. (In the plane its boundary consists of two sets of vertical semi-infinite lines.) It characterizes, in terms of the spacing between the hyperplanes, those domains for which there exist minimal harmonic functions with a certain exponential growth.
\end{abstract}

\section{Introduction}

The subtle relationship between the structure of positive harmonic functions on a domain $\Omega$ in $\mathbf{R}^{N}(N \geq 2)$ and boundary geometry has been much studied. One avenue of investigation has been to examine the effect of modifying the boundary of a familiar domain such as a half-space, cone or cylinder. Thus many authors have been led to investigate the case of Denjoy domains $\Omega$, where the complement $\mathbf{R}^{N} \backslash \Omega$ is contained in a hyperplane, say $\mathbf{R}^{N-1} \times\{0\}($ see $[6,11,14,1,24,25,8,10,2,21])$. For example, Benedicks [6] has established a harmonic measure criterion that describes when the cone of positive harmonic functions on $\Omega$ that vanish on the boundary $\partial \Omega$ is generated by two linearly independent minimal harmonic functions. (We recall that a positive harmonic function $h$ on a domain $\Omega$ is called minimal if any nonnegative harmonic minorant of $h$ on $\Omega$ is proportional to $h$.) Benedicks' criterion is also equivalent to the existence of a harmonic function $u$ on $\Omega$ vanishing on $\partial \Omega$ and satisfying $u(x) \geq\left|x_{N}\right|$ on $\Omega$, and thus describes when a Denjoy domain behaves like the union of two half-spaces from the point of view of potential theory. Related results, based on sectors, cones or cylinders, may be found in $[12,21,18]$. The purpose of this paper is to describe what happens in the case of another relative of the infinite cylinder. More precisely, let $\left(a_{n}\right)$ be a strictly increasing sequence of non-negative numbers such that $a_{n} \rightarrow+\infty$ and $a_{n+1}-a_{n} \rightarrow 0$ as $n \rightarrow \infty$, and let $B^{\prime}$ be the unit ball in $\mathbf{R}^{N-1}$. We define

$$
E=\bigcup_{n \in \mathbf{N}}\left(\mathbf{R}^{N-1} \backslash B^{\prime}\right) \times\left\{a_{n}\right\}
$$

and investigate when the domain $\Omega=\mathbf{R}^{N} \backslash E$ inherits the potential theoretic character of the cylinder $U=B^{\prime} \times \mathbf{R}$; that is, when the set $E$ imitates $\partial U$ in terms of its effect on the asymptotic behaviour of positive harmonic functions on $\Omega$. We call

doi:10.5186/aasfm.2011.3630

2010 Mathematics Subject Classification: Primary 31B25, 31C35.

Key words: Minimal harmonic functions, comb-like domain, harmonic measure.

This research was supported by Science Foundation Ireland under Grant/RFP/MAT057, and is also part of the programme of the ESF Network "Harmonic and Complex Analysis and Applications" (HCAA). 
such domains $\Omega$ comb-like because they are a generalization of comb domains in the plane.

Let $x=\left(x^{\prime}, x_{N}\right)$ denote a typical point of Euclidean space $\mathbf{R}^{N}=\mathbf{R}^{N-1} \times \mathbf{R}$. It is known (see [15], for example) that the cone of positive harmonic functions on $U$ that vanish on $\partial U$ is generated by two minimal harmonic functions $h_{ \pm}\left(x^{\prime}, x_{N}\right)=$ $e^{ \pm \alpha x_{N}} \phi\left(x^{\prime}\right)$, where $\alpha$ is the square root of the first eigenvalue of the operator $-\Delta=$ $-\sum_{j=1}^{N-1} \partial^{2} / \partial x_{j}^{2}$ on $B^{\prime}$ and $\phi$ is the corresponding eigenfunction, normalized by $\phi(0)=$ 1. We describe below when a comb-like domain admits a minimal harmonic function $u$ that vanishes on $\partial \Omega$ and satisfies $u \geq h_{+}$on $U$.

Theorem 1.1. Let $\nu>1$. Assume that $\left(a_{n}\right)$ satisfies the following condition

$$
\frac{1}{\nu} \leq \frac{a_{k+1}-a_{k}}{a_{j+1}-a_{j}} \leq \nu
$$

whenever $\left|a_{k}-a_{j}\right|<4$. The following statements are equivalent:

(a) there exists a positive harmonic function $u$ on $\Omega$ that satisfies $u \geq h_{+}$on $U$ and $u$ vanishes continuously on $E$;

(b) $\sum_{n=1}^{\infty}\left(a_{n+1}-a_{n}\right)^{2}<+\infty$.

Moreover, if (b) holds, then $u$ can be chosen to be minimal in part (a).

We will prove Theorem 1.1 by combining methods from [14], [12] and [18] with some new ideas. It is known (see $[7,9,16])$ that the behaviour of minimal harmonic functions on simply connected domains is intimately related to the classical angular derivative problem. We note that when $N=2$, condition (b) of Theorem 1.1 is necessary and sufficient for the comb domain $\Omega$ to have an angular derivative at $+\infty$ (see $[22,23,20])$.

\section{Notation and preliminary results}

We use $\partial^{\infty} D$ to denote the boundary of a domain $D$ in compactified space $\mathbf{R}^{N} \cup$ $\{\infty\}$. Let $B_{\rho}(x)$ denote the open ball in $\mathbf{R}^{N}$ of centre $x$ and radius $\rho>0$. We write $B_{\rho}^{\prime}$ (resp. $B_{\rho}$ ) for the open ball in $\mathbf{R}^{N-1}\left(\operatorname{resp} . \mathbf{R}^{N}\right)$ of centre 0 and radius $\rho$, and $V(\rho)=\partial B_{\rho}^{\prime} \times \mathbf{R}$. If $\rho=1$, we write $B^{\prime}$ instead of $B_{1}^{\prime}$. For $0<\rho_{1}<\rho_{2}$ let $A\left(\rho_{1}, \rho_{2}\right)=\left(B_{\rho_{2}}^{\prime} \backslash \overline{B_{\rho_{1}}^{\prime}}\right) \times \mathbf{R}$. We denote by $\mu_{x}^{D}$ the harmonic measure for an open set $D \subset \mathbf{R}^{N}$ evaluated at $x \in D$. If $f$ is a function defined on $\partial^{\infty} D$, we use $\bar{H}_{f}^{D}$ to denote the upper Perron-Wiener-Brelot solution to the Dirichlet problem on $D$ and $H_{f}^{D}$ for the PWB solution of the Dirichlet problem on $D$ when it exists. We denote by $P_{D}(\cdot, y)$ the Poisson kernel for $D$ with pole $y \in \partial D$, where $\partial D$ is smooth enough for it to be defined. If $W \subseteq D$ and $u$ is a superharmonic function on $D$, we denote by $R_{u}^{W}$ (resp. $\widehat{R}_{u}^{W}$ ) the reduced function (resp. the regularized reduced function) of $u$ relative to $W$ in $D$. We denote surface area measure on a given surface by $\sigma$. We use $C(a, b, \ldots)$ to denote a constant depending at most on $a, b, \ldots$, the value of which may change from line to line.

For the remainder of the paper, we fix $0<r<1<R$ and for $x \in \partial U$ we define $F_{x}=\partial B_{r}^{\prime} \times\left[x_{N}-1, x_{N}+1\right]$ and

$$
T_{x}=\left(B_{R}^{\prime} \backslash \overline{B_{r}^{\prime}}\right) \times\left(x_{N}-1, x_{N}+1\right) .
$$


We note that the first eigenfunction $\phi$ of $-\Delta$ in $B^{\prime}$ is comparable with the distance to $\partial B^{\prime}$, that is

$$
C_{1}(N)\left(1-\left|x^{\prime}\right|\right) \leq \phi\left(x^{\prime}\right) \leq C_{2}(N)\left(1-\left|x^{\prime}\right|\right) \quad\left(x^{\prime} \in B^{\prime}\right) .
$$

A simple proof of $(2.1)$ can be found in [17, pp. 419-420]. The following estimate for the Poisson kernel (see [18, Section 2.1], for example) will prove useful. For $\left|x^{\prime}\right|=s<1, x_{N} \in \mathbf{R}, y \in \partial U$

$$
C_{1}(N, s) e^{-\alpha\left|x_{N}-y_{N}\right|} \leq P_{U}(x, y) \leq C_{2}(N, s) e^{-\alpha\left|x_{N}-y_{N}\right|} .
$$

If $0<r_{1}<s<r_{2}$, similar estimates hold for $P_{A\left(r_{1}, r_{2}\right)}$ with $\alpha$ replaced by the square root of the first eigenvalue of $-\Delta$ in $B_{r_{2}}^{\prime} \backslash \overline{B_{r_{1}}^{\prime}}$ and constants $C_{1}, C_{2}$ depending on $N, r_{1}, r_{2}$ and $s$.

Proposition 2.1. Assume there exists a positive harmonic function $u$ on $\Omega$ such that $u \geq h_{+}$on $U$ and $u$ vanishes on $E$. Then

$$
\sum_{n=1}^{\infty}\left(a_{n+1}-a_{n}\right)^{2}<+\infty
$$

Proof. By (2.2) we have

$$
\begin{aligned}
+\infty & >u(0) \geq \int_{\partial U} u(y) P_{U}(0, y) d \sigma(y) \\
& \geq C(N) \sum_{n=1}^{\infty} \int_{\partial B^{\prime} \times\left(a_{n}, a_{n+1}\right)} u(y) e^{-\alpha y_{N}} d \sigma(y) .
\end{aligned}
$$

We use Harnack's inequalities and (2.1) to see that for $y \in \partial U$ with

$$
y_{N} \in\left(a_{n}+\left(a_{n+1}-a_{n}\right) / 4, a_{n+1}-\left(a_{n+1}-a_{n}\right) / 4\right)
$$

the following holds

$$
\begin{aligned}
u(y) & \geq C(N) u\left(\left(1-\left(a_{n+1}-a_{n}\right) / 8\right) y^{\prime}, y_{N}\right) \\
& \geq C(N) e^{\alpha y_{N}} \phi\left(\left(1-\left(a_{n+1}-a_{n}\right) / 8\right) y^{\prime}\right) \\
& \geq C(N) e^{\alpha y_{N}}\left(a_{n+1}-a_{n}\right) .
\end{aligned}
$$

We deduce from (2.4) and (2.5) that (2.3) holds.

Assume now that $\sum_{n=1}^{\infty}\left(a_{n+1}-a_{n}\right)^{2}<+\infty$. Let $J \in \mathbf{N}$ be large enough so that $a_{n+1}-a_{n} \leq 1 / 2$ for $n \geq J$. For ease of exposition we rename the sequence $\left(a_{n}\right)_{n=J}^{\infty}$ as $\left(b_{n}\right)_{n=1}^{\infty}$. We also define $\rho_{n}=\left(b_{n+1}-b_{n}\right) / 2$ for $n \in \mathbf{N}$. We introduce $b_{0}=b_{1}-1$ and $\rho_{0}=1 / 2$. For technical reasons, we will work with

$$
E^{\prime \prime}=\bigcup_{n=1}^{\infty}\left(\mathbf{R}^{N-1} \backslash B^{\prime}\right) \times\left\{b_{n}\right\} \quad \text { and } \quad E^{\prime}=\left(\partial B^{\prime} \times\left(-\infty, b_{1}\right]\right) \cup E^{\prime \prime},
$$

and at the end we will dispense with these additional requirements.

Lemma 2.1. There exists a positive constant $c_{1}$, depending on $N, R$ and $r$, such that for any $x \in \partial U$ we have

$$
\mu_{x}^{T_{x} \backslash E^{\prime \prime}}\left(F_{x}\right) \leq \mu_{x}^{T_{x} \backslash E^{\prime \prime}}\left(\partial T_{x}\right) \leq c_{1} \mu_{x}^{T_{x} \backslash E^{\prime \prime}}\left(F_{x}\right) .
$$


Proof. Let $x \in \partial U$. The left hand inequality in (2.6) is obvious since $F_{x} \subset \partial T_{x}$. Let $h=H_{\chi_{x}}^{T_{x}}$ on $T_{x}$ and $h=\chi_{F_{x}}$ on $\partial T_{x}$. In order to establish the right hand inequality, it is enough to prove that

$$
h \leq h(x) \text { on } E^{\prime \prime} \cap T_{x} .
$$

We will borrow an argument from [18, Lemma 2.1]. Using reflection in $\mathbf{R}^{N-1} \times\left\{x_{N}+\right.$ $1\}$ to extend $h$ to $\left(\overline{B_{R}^{\prime}} \backslash B_{r}^{\prime}\right) \times\left[x_{N}-1, x_{N}+3\right]$, and translation, for $y \in \partial B^{\prime} \times\left(x_{N}, x_{N}+1\right)$ we obtain

$$
\begin{aligned}
h(y) & =H_{h}^{T_{x}+\left(0^{\prime}, y_{N}-x_{N}\right)}(y) \\
& =\mu_{x}^{T_{x}}\left(\partial B_{r}^{\prime} \times\left[x_{N}-1,2 x_{N}+1-y_{N}\right]\right)-\mu_{x}^{T_{x}}\left(\partial B_{r}^{\prime} \times\left[2 x_{N}+1-y_{N}, x_{N}+1\right]\right) \\
& \leq \mu_{x}^{T_{x}}\left(F_{x}\right)=h(x) .
\end{aligned}
$$

By symmetry, $h(y) \leq h(x)$ for $y \in \partial B^{\prime} \times\left(x_{N}-1, x_{N}+1\right)$. Since

$h(y)=0 \leq h(x)$ for $y \in\left[\partial B_{R}^{\prime} \times\left(x_{N}-1, x_{N}+1\right)\right] \cup\left[\left(B_{R}^{\prime} \backslash \overline{B^{\prime}}\right) \times\left\{x_{N}-1, x_{N}+1\right\}\right]$,

using the maximum principle, we see that $h \leq h(x)$ on $\left(B_{R}^{\prime} \backslash \overline{B^{\prime}}\right) \times\left(x_{N}-1, x_{N}+1\right)$, which proves (2.7).

We note that Lemma 2.1 holds in a more general context when $E^{\prime \prime}$ is a closed subset of $\mathbf{R}^{N} \backslash U$.

To prove Theorem 1.1 we shall need the following estimate.

Lemma 2.2. Let $\nu>1$. Assume that $\left(b_{n}\right)$ satisfies

$$
\frac{1}{\nu} \leq \frac{b_{k+1}-b_{k}}{b_{j+1}-b_{j}} \leq \nu
$$

whenever $\left|b_{k}-b_{j}\right|<4$. Then there exists a constant $c_{2}$, depending only on $N, r$ and $\nu$, such that

$$
\mu_{x}^{T_{x} \backslash E^{\prime \prime}}\left(F_{x}\right) \leq c_{2}\left(b_{n+1}-b_{n}\right)
$$

whenever $x \in \partial B^{\prime} \times\left(b_{n}, b_{n+1}\right)$ and $n \in \mathbf{N}$.

Proof. We suppose that $x \in \partial B^{\prime} \times\left(b_{n}, b_{n+1}\right)$ for some $n \in \mathbf{N}$. We define $\omega=$ $\left(B_{R}^{\prime} \backslash \overline{B_{r}^{\prime}}\right) \times\left(b_{j_{0}}, b_{k_{0}}\right)$, where $j_{0}=\max \left\{j: b_{j} \leq x_{N}-1\right\}$ and $k_{0}=\min \left\{j: b_{j} \geq x_{N}+1\right\}$. Let $g=H_{\chi_{V(r)}}^{\omega \backslash E^{\prime \prime}}$ on $\omega \backslash E^{\prime \prime}$ and define $g=\chi_{V(r)}$ elsewhere. Let $m=\sup _{\partial U} g$. We note that

$$
\mu_{x}^{T_{x} \backslash E^{\prime \prime}}\left(F_{x}\right) \leq \mu_{x}^{\omega \backslash E^{\prime \prime}}(V(r)) \leq m .
$$

We will obtain an upper bound for $m$ in terms of $\rho_{n}$. To do this, we define an open set $Z$ as follows

$$
Z=\omega \backslash \bigcup_{k=0}^{\infty} \bigcup_{p \in\left[b_{k}, b_{k+1}\right]}\left\{z \in \overline{B_{s}^{\prime}} \times\{p\}: s=(1-r)\left(\left|p-\left(b_{k}+\rho_{k}\right)\right|-\rho_{k}\right)+1\right\} .
$$

We estimate $g$ on $\partial Z$ in terms of $m$ and $\rho_{n}$. Since $g=0$ on $\partial Z \backslash U$, we estimate $g$ on $\partial Z \cap U$, noting that, for $y \in \omega \cap U$, we have

$$
g(y)=H_{g}^{\omega \cap U}(y)=H_{\chi_{V(r)}}^{\omega \cap U}(y)+\int_{\partial U \cap \omega} g d \mu_{y}^{\omega \cap U} .
$$


Let $g_{1}(y)=H_{\chi_{V(r)}}^{\omega \cap U}(y)$ and $g_{2}(y)=\int_{\partial U \cap \omega} g d \mu_{y}^{\omega \cap U}$ for $y \in \omega \cap U$. Using the function

$$
f_{N}(y)= \begin{cases}\left|y^{\prime}\right|^{3-N}-1 & (N \geq 4) \\ -\log \left|y^{\prime}\right| & (N=3) \\ 1-\left|y^{\prime}\right| & (N=2)\end{cases}
$$

and the maximum principle, we find that for $y \in \partial Z \cap U$

$$
g_{1}(y) \leq f_{N}(y) / f_{N}(r x) \leq C_{1}(N, r)\left(1-\left|y^{\prime}\right|\right) \leq C_{1}(N, r, \nu) \rho_{n} .
$$

We now wish to show that there exists a constant $C_{2}(N, \nu) \in(0,1)$ such that

$$
g_{2} \leq C_{2}(N, \nu) m \text { on } \partial Z \cap U \text {. }
$$

Let $l=(1-r) \min _{j_{0} \leq k \leq k_{0}-1} \rho_{k}$ and let $t=\left(1,0, \ldots, 0, t_{N}\right)$ with $t_{N} \in\left\{b_{k}: k=\right.$ $\left.j_{0}+1, \ldots, k_{0}-1\right\}$. By [5, Lemma 8.5.1], for $x \in B_{l / 2}\left(1+l, 0, \ldots, 0, t_{N}\right)$ we have $g(x) \leq$ $C(N)\left(g\left(p_{+}\right)+g\left(p_{-}\right)\right)$, where $p_{ \pm}=\left(1+l, 0, \ldots, 0, t_{N} \pm l / 2\right)$. Using a Harnack chain to cover the longer arc joining $p_{+}$and $p_{-}$along the circle $\partial B_{\sqrt{5} l / 2}(t) \cap\left(\mathbf{R} \times\{0\}^{N-2} \times \mathbf{R}\right)$, we deduce that $g \leq C_{3}(N) m$ on that circle. By the invariance of $g$ under rotations around the $x_{N}$-axis and the maximum principle, this inequality holds on a torusshaped set enclosing the edge of $\left(\mathbf{R}^{N-1} \backslash B^{\prime}\right) \times\left\{t_{N}\right\}$; more precisely on every closed ball centred at a point of $\partial B^{\prime} \times\left\{t_{N}\right\}$ and having radius $\sqrt{5} l / 2$. When $t_{N}=b_{j_{0}}$ or $t_{N}=b_{k_{0}}$, this inequality follows directly from [5, Lemma 8.5.1], with a perhaps different constant, $C_{4}(N)$ say. In particular, for $y \in B^{t} \backslash E^{t}$, where $B^{t}=B_{\sqrt{5} l / 2}(t)$, $E^{t}=[1,+\infty) \times \mathbf{R}^{N-2} \times\left\{t_{N}\right\}$ and $t_{N} \in\left\{b_{k}: k=j_{0}, \ldots, k_{0}\right\}$, we have

$$
g(y) \leq H_{g}^{B^{t} \backslash E^{t}}(y)=\int_{\partial B^{t}} g d \mu_{y}^{B^{t} \backslash E^{t}} \leq \max \left\{C_{3}(N), C_{4}(N)\right\} m H_{\chi_{\partial B^{t}}}^{B^{t}}(y) .
$$

Since $t$ is a regular boundary point for $B^{t} \backslash E^{t}$, there exists $\delta=\delta(N)>0$ such that

$$
H_{\chi_{\partial B^{t}}}^{B^{t} \backslash E^{t}}(y) \leq \frac{1}{2 \max \left\{C_{3}(N), C_{4}(N)\right\}} \quad\left(y \in B_{\delta l}(t) \backslash E^{t}\right) .
$$

Let $K_{\delta l}=\bigcup_{k=j_{0}}^{k_{0}}\left\{y \in \partial U:\left|y_{N}-b_{k}\right|<\delta l\right\}$. In view of (2.12) and (2.13), and the invariance of $g$ under rotations around the $x_{N}$-axis, we conclude that $g \leq m / 2$ on $K_{\delta l}$.

Hence, for $y \in \partial Z \cap U$, we have

$$
g_{2}(y) \leq \int_{\partial U} g d \mu_{y}^{U} \leq \frac{m}{2} \mu_{y}^{U}\left(K_{\delta l}\right)+m \mu_{y}^{U}\left(\partial U \backslash K_{\delta l}\right) \leq m\left(1-\frac{1}{2} \mu_{y}^{U}\left(K_{\delta l}\right)\right) .
$$

We now show that there exists a constant $C_{5}(N, \nu) \in(0,1)$ such that $\mu_{y}^{U}\left(K_{\delta l}\right) \geq$ $C_{5}(N, \nu)$ for $y \in \partial Z \cap U$. We first estimate $\mu_{y}^{U}\left(K_{\delta l}\right)$ on some ball centred at $t$ and then join other points of $\partial Z \cap U$ by a Harnack chain.

Let $W_{\delta l}=B^{\prime} \times\left(t_{N}-\delta l, t_{N}+\delta l\right)$. We use a dilation $\psi(y)=t+(y-t) /(\delta l)$ and note that, by continuity, there exists an absolute positive constant $\gamma$ such that for $y \in \psi\left(W_{\delta l}\right) \cap B_{\gamma}(t)$ the following inequalities hold

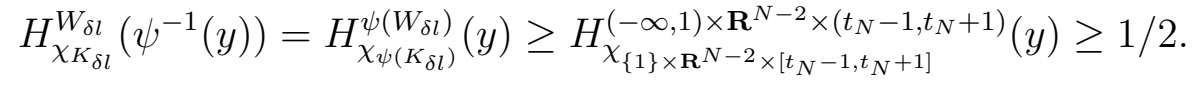

Hence

$$
\mu_{y}^{U}\left(K_{\delta l}\right) \geq \mu_{y}^{W_{\delta l}}\left(K_{\delta l}\right) \geq 1 / 2 \quad\left(y \in B_{\gamma \delta l}(t) \cap U\right)
$$


and by Harnack's inequalities

$$
\mu_{y}^{U}\left(K_{\delta l}\right) \geq C_{5}(N, \nu) \text { for all } y \in \partial Z \cap U .
$$

Let $C_{2}(N, \nu)=1-C_{5}(N, \nu) / 2$. Then (2.11) holds in view of (2.14), and by (2.9) and (2.10) we have

$$
g \leq C_{1}(N, r, \nu) \rho_{n}+C_{2}(N, \nu) m \text { on } \partial Z .
$$

By the maximum principle this inequality holds on $Z$ and implies that

$$
m \leq \frac{C_{1}(N, r, \nu)}{1-C_{2}(N, \nu)} \rho_{n}
$$

This finishes the proof of lemma.

We define $\beta_{E^{\prime}}(x)$ to be the harmonic measure of $\partial T_{x}$ in $T_{x} \backslash E^{\prime}$ evaluated at $x$. If $x \in E^{\prime}$, then $\beta_{E^{\prime}}(x)$ is interpreted as 0 . We observe that, if $\left(b_{n}\right)$ satisfies the ratio condition (2.8), then, in view of Lemmas 2.1 and 2.2, we have

$$
\begin{aligned}
\int_{\partial B^{\prime} \times\left(b_{1},+\infty\right)} \beta_{E^{\prime}}(y) d \sigma(y) & \leq c_{1} \int_{\partial B^{\prime} \times\left(b_{1},+\infty\right)} \mu_{y}^{T_{y} \backslash E^{\prime \prime}}\left(F_{y}\right) d \sigma(y) \\
& \leq c_{1} c_{2} \sigma_{N-1} \sum_{n=1}^{\infty}\left(b_{n+1}-b_{n}\right)^{2}
\end{aligned}
$$

where $\sigma_{N-1}$ denotes the surface measure of $\partial B^{\prime}$ in $\mathbf{R}^{N-1}$.

Henceforth let $\left(b_{n}\right)$ satisfy $(2.8)$ and let

$$
\Lambda=\sum_{n=1}^{\infty}\left(b_{n+1}-b_{n}\right)^{2}<+\infty .
$$

Before we prove the next lemma, we collect together some facts about certain Bessel functions (see [4, Section 4]). Let $K=K_{(N-3) / 2}:(0, \infty) \rightarrow(0, \infty)$ denote the Bessel function of the third kind, of order $(N-3) / 2$. Then the function

$$
h_{0}\left(x^{\prime}, x_{N}\right)=\left|x^{\prime}\right|^{(3-N) / 2} K\left(\pi\left|x^{\prime}\right|\right) \sin \left(\pi x_{N}\right)
$$

is positive and superharmonic on the strip $\mathbf{R}^{N-1} \times(0,1)$, harmonic on $\left(\mathbf{R}^{N-1} \backslash\left\{0^{\prime}\right\}\right) \times$ $(0,1)$ and vanishes on $\mathbf{R}^{N-1} \times\{0,1\} \backslash\left\{\left(0^{\prime}, 0\right),\left(0^{\prime}, 1\right)\right\}$. Moreover, there exists $c(N) \geq 1$ such that

$$
c(N)^{-1} \leq(2 t / \pi)^{1 / 2} e^{t} K(t) \leq c(N) \text { for } t \in[1,+\infty) .
$$

We also recall a result of Domar ([13, Theorem 2]). Suppose that $D$ is a domain in $\mathbf{R}^{N}$ and $F: D \rightarrow[0,+\infty]$ is a given upper semicontinuous function on $D$. Let $\mathcal{F}$ be the collection of all subharmonic functions $u$, such that $u \leq F$ on $D$. Domar's result says that if

$$
\int_{D}\left[\log ^{+} F(x)\right]^{N-1+\varepsilon} d x<\infty,
$$

for some $\varepsilon>0$, then the function $M(x)=\sup _{u \in \mathcal{F}} u(x)$ is bounded on every compact subset of $D$.

Let $0<r^{\prime}<\min \{r, 1 / 2\}$. Define $V=A\left(r^{\prime}, \infty\right) \backslash E^{\prime}$ and $U_{n}=\left(\mathbf{R}^{N-1} \backslash \overline{B^{\prime}}\right) \times$ $\left(b_{n}, b_{n+1}\right)$ for $n \in \mathbf{N}$. 
Lemma 2.3. There exists a positive constant $c_{3}$, depending on $N, R, r$ and $r^{\prime}$, such that, for any positive harmonic function $u$ on $V$ that is bounded on each $U_{n}$ and vanishes on $E^{\prime}$,

$$
u(y) \leq c_{3} u\left(r x^{\prime}, x_{N}\right) H_{\chi \partial T_{x}}^{T_{x} \backslash E^{\prime}}(y) \quad\left(x \in \partial U, y \in T_{x} \backslash E^{\prime}\right) .
$$

In particular,

$$
u\left(x^{\prime}, x_{N}\right) \leq c_{3} \beta_{E^{\prime}}(x) u\left(r x^{\prime}, x_{N}\right) \quad(x \in \partial U) .
$$

Proof. Let $x \in \partial U, l=\left(1+r^{\prime}\right) / 3$ and $L=2 R$. Define $A_{x}=\left\{y: l<\left|y^{\prime}\right|<\right.$ $\left.L,\left|x_{N}-y_{N}\right|<2\right\}$. We will show that

$$
\frac{u(y)}{C\left(N, r, r^{\prime}\right) u\left(r x^{\prime}, x_{N}\right)} \leq F(y) \quad\left(y \in A_{x}\right),
$$

where

$$
F(y)= \begin{cases}|1-| y^{\prime}||^{1-N}, & \left|y^{\prime}\right| \neq 1 \\ +\infty, & \left|y^{\prime}\right|=1 .\end{cases}
$$

Step 1. Let $\left(y^{\prime}, y_{N}\right) \in A_{x} \cap U$. Harnack's inequalities yield that

$$
u(y) \leq C\left(N, r, r^{\prime}\right) u\left(r x^{\prime}, x_{N}\right)\left(1-\left|y^{\prime}\right|\right)^{1-N} .
$$

Step 2. If $y \in A_{x} \cap U_{n}$ and $\left|y^{\prime}\right|-1 \leq \min \left\{y_{N}-b_{n}, b_{n+1}-y_{N}\right\}$, then there is a Harnack chain of fixed length joining $\left(y^{\prime}, y_{N}\right)$ with $\left(\left(2-\left|y^{\prime}\right|\right) y^{\prime} /\left|y^{\prime}\right|, y_{N}\right) \in A_{x} \cap U$. By Step 1, we have

$$
u(y) \leq C(N) u\left(\left(2-\left|y^{\prime}\right|\right) y^{\prime} /\left|y^{\prime}\right|, y_{N}\right) \leq C\left(N, r, r^{\prime}\right) u\left(r x^{\prime}, x_{N}\right)\left(\left|y^{\prime}\right|-1\right)^{1-N} .
$$

Step 3. If $y \in A_{x} \cap U_{n}$ and $\rho_{n} \geq\left|y^{\prime}\right|-1>\min \left\{y_{N}-b_{n}, b_{n+1}-y_{N}\right\}$, we apply [5, Lemma 8.5.1] and Harnack's inequalities to see that

$$
u(y) \leq C(N) u\left(y^{\prime}, \widetilde{y}_{N}\right),
$$

where $\widetilde{y}_{N}$ is such that $\left|\widetilde{y}_{N}-y_{N}\right|<\left|b_{n}+\rho_{n}-y_{N}\right|$ and $\left|y^{\prime}\right|-1=\min \left\{\widetilde{y}_{N}-b_{n}, b_{n+1}-\widetilde{y}_{N}\right\}$. By Step 2,

$$
u(y) \leq C\left(N, r, r^{\prime}\right) u\left(r x^{\prime}, x_{N}\right)\left(\left|y^{\prime}\right|-1\right)^{1-N} .
$$

Step 4. If $y \in A_{x} \cap U_{n}$ and $\left|y^{\prime}\right| \geq 1+\rho_{n}$, let $V_{n}=\left\{\left(z^{\prime}, z_{N}\right): 1+\rho_{n}<\left|z^{\prime}\right|, z_{N} \in\right.$ $\left.\left(b_{n}, b_{n+1}\right)\right\}$. For $z \in U_{n}$ we define a function

$$
h_{n}(z)=\frac{h_{0}\left(\left(z^{\prime}, z_{N}-b_{n}\right) /\left(2 \rho_{n}\right)\right)}{K\left(\pi\left(1+\rho_{n}\right) /\left(2 \rho_{n}\right)\right)}\left(\frac{1+\rho_{n}}{2 \rho_{n}}\right)^{(N-3) / 2}
$$

which is harmonic on $U_{n}$ and vanishes on $\partial U_{n} \backslash \partial U$. Applying [5, Lemma 8.5.1] and Harnack's inequalities to $u$ and $h_{n}$, by Step 3, we get

$$
u(z) \leq C\left(N, r, r^{\prime}\right) u\left(r x^{\prime}, x_{N}\right) \rho_{n}^{1-N} h_{n}(z) \text { for } z \in \partial V_{n} .
$$

Since $u$ is bounded on $V_{n}$ and $\infty$ has zero harmonic measure for $V_{n}$,

$$
u(y) \leq C\left(N, r, r^{\prime}\right) u\left(r x^{\prime}, x_{N}\right) \rho_{n}^{1-N} h_{n}(y) .
$$

Furthermore, by (2.16) and (2.17)

$$
\begin{aligned}
h_{n}(y) & \leq\left(\frac{1+\rho_{n}}{\left|y^{\prime}\right|}\right)^{\frac{N-3}{2}} K\left(\frac{\pi\left|y^{\prime}\right|}{2 \rho_{n}}\right)\left(K\left(\frac{\pi\left(1+\rho_{n}\right)}{2 \rho_{n}}\right)\right)^{-1} \\
& \leq C(N) e^{-\frac{\pi}{2 \rho_{n}}\left(\left|y^{\prime}\right|-1\right)}\left(\frac{1+\rho_{n}}{\left|y^{\prime}\right|}\right)^{\frac{N-2}{2}} \leq C(N) e^{-\frac{\pi}{2 \rho_{n}}\left(\left|y^{\prime}\right|-1\right)} \leq C(N)\left(\frac{\left|y^{\prime}\right|-1}{\rho_{n}}\right)^{1-N} .
\end{aligned}
$$


Hence we see from (2.20) that

$$
u(y) \leq C\left(N, r, r^{\prime}\right) u\left(r x^{\prime}, x_{N}\right)\left(\left|y^{\prime}\right|-1\right)^{1-N} .
$$

We conclude that (2.19) follows from Steps 1-4. Since

$$
\int_{A_{x}}\left(\log ^{+} F(y)\right)^{N} d y \leq C(N, R)
$$

Domar's result and Harnack's inequalities (if $r<l$ ) yield

$$
u(y) \leq C\left(N, R, r, r^{\prime}\right) u\left(r x^{\prime}, x_{N}\right) \quad\left(y \in \overline{T_{x}}\right) .
$$

Therefore

$$
u(y)=H_{u}^{T_{x} \backslash E^{\prime}}(y) \leq C\left(N, R, r, r^{\prime}\right) u\left(r x^{\prime}, x_{N}\right) H_{\chi_{\partial T_{x}}}^{T_{x} \backslash E^{\prime}}(y) \quad\left(y \in T_{x} \backslash E^{\prime}\right) .
$$

In particular,

$$
u(x) \leq C\left(N, R, r, r^{\prime}\right) u\left(r x^{\prime}, x_{N}\right) \beta_{E^{\prime}}(x) .
$$

Lemma 2.4. Let $v: \mathbf{R}^{N} \cup\{\infty\} \rightarrow[0,+\infty]$ be a Borel measurable function such that $v(x) \leq e^{\alpha x_{N}} \chi_{V\left(r^{\prime}\right)}(x)$ on $\mathbf{R}^{N}$. There exist positive constants $c_{4}$ and $c_{5}$, depending on $N, R, r$ and $r^{\prime}$, such that, if $\Lambda \leq c_{4}$, then $H_{v}^{V}$ exists and

$$
H_{v}^{V}(x) \leq H_{v}^{A\left(r^{\prime}, 1\right)}(x)+c_{5} \Lambda e^{\alpha x_{N}} \quad\left(\left|x^{\prime}\right|=r\right) .
$$

Proof. Let $h_{n}=H_{\min \{v, n\}}^{V}$ on $V$ and $h_{n}=\min \{v, n\}$ on $\partial^{\infty} V$, and let

$$
m_{n}=\sup \left\{e^{-\alpha x_{N}} h_{n}\left(x^{\prime}, x_{N}\right):\left|x^{\prime}\right|=r, x_{N}>-n\right\} .
$$

Then

$$
h_{n}=H_{h_{n}}^{A\left(r^{\prime}, 1\right)}=H_{h_{n} \chi \partial U}^{A\left(r^{\prime}, 1\right)}+H_{h_{n} \chi_{V\left(r^{\prime}\right)}}^{A\left(r^{\prime}, 1\right)} \quad \text { in } A\left(r^{\prime}, 1\right) .
$$

Let $\alpha_{r^{\prime}}>0$ denote the square root of the first eigenvalue of $-\Delta$ in $B^{\prime} \backslash \overline{B_{r^{\prime}}^{\prime}}$. Then $\alpha<\alpha_{r^{\prime}}$ because the complement of $B^{\prime} \backslash \overline{B_{r^{\prime}}^{\prime}}$ in $B^{\prime}$ is non-polar (see [19, Section 1.3.2]). Since $d \mu_{x}^{A\left(r^{\prime}, 1\right)}=P_{A\left(r^{\prime}, 1\right)}(x, \cdot) d \sigma$ on $\partial U$, the Poisson kernel estimates yield, for $\left|x^{\prime}\right|=r$, that

$$
\begin{aligned}
e^{-\alpha x_{N}} H_{h_{n} \chi \partial U}^{A\left(r^{\prime}, 1\right)}(x) & \leq C\left(N, r, r^{\prime}\right) e^{-\alpha x_{N}} \int_{\partial U} h_{n}(y) e^{-\alpha_{r^{\prime}}\left|x_{N}-y_{N}\right|} d \sigma(y) \\
& \leq C\left(N, r, r^{\prime}\right) \int_{\partial U} h_{n}(y) e^{-\alpha y_{N}} d \sigma(y) .
\end{aligned}
$$

Noting that $h_{n}$ satisfies the hypotheses of Lemma 2.3, we see from (2.15) that, when $\left|x^{\prime}\right|=r$ we have

$$
\begin{aligned}
e^{-\alpha x_{N}} H_{h_{n} \chi \partial U}^{A\left(r^{\prime}, 1\right)}(x) & \leq C\left(N, R, r, r^{\prime}\right) \int_{\partial U} e^{-\alpha y_{N}} h_{n}\left(r y^{\prime}, y_{N}\right) \beta_{E^{\prime}}(y) d \sigma(y) \\
& \leq C_{1} m_{n} \Lambda
\end{aligned}
$$

where $C_{1}$ is a constant depending on $N, R, r, r^{\prime}$ and $\nu$. 
Moreover, for $\left|x^{\prime}\right|=r$ we have

$$
\begin{aligned}
e^{-\alpha x_{N}} H_{h_{n} \chi_{V\left(r^{\prime}\right)}}^{A\left(r^{\prime}, 1\right)}(x) & \leq e^{-\alpha x_{N}} \int_{V\left(r^{\prime}\right)} e^{\alpha y_{N}} d \mu_{x}^{A\left(r^{\prime}, 1\right)}(y) \\
& \leq C\left(N, r, r^{\prime}\right) \int_{V\left(r^{\prime}\right)} e^{\alpha\left(y_{N}-x_{N}\right)} e^{-\alpha_{r^{\prime}}\left|y_{N}-x_{N}\right|} d \sigma(y) \\
& \leq C\left(N, r, r^{\prime}\right) \int_{-\infty}^{+\infty} e^{\left(\alpha-\alpha_{r^{\prime}}\right)\left|y_{N}-x_{N}\right|} d y_{N} \leq C_{2}\left(N, r, r^{\prime}\right)
\end{aligned}
$$

By (2.21)-(2.23) we obtain

$$
e^{-\alpha x_{N}} h_{n}(x)=e^{-\alpha x_{N}} H_{h_{n} \chi_{\partial U}}^{A\left(r^{\prime}, 1\right)}(x)+e^{-\alpha x_{N}} H_{h_{n} \chi_{V\left(r^{\prime}\right)}}^{A\left(r^{\prime}, 1\right)}(x) \leq C_{1} m_{n} \Lambda+C_{2} \quad\left(\left|x^{\prime}\right|=r\right) .
$$

Taking $c=\max \left\{C_{1}, C_{2}\right\}$ we arrive at

$$
m_{n} \leq c\left(1+m_{n} \Lambda\right) .
$$

We choose $c_{4}=(2 c)^{-1}$ and suppose that $\Lambda \leq c_{4}$. Then

$$
m_{n} \leq c+m_{n} c c_{4}=c+m_{n} / 2
$$

which implies that $m_{n} \leq 2 c$.

It follows from (2.21) and (2.22) that for $\left|x^{\prime}\right|=r$ we have

$$
e^{-\alpha x_{N}} h_{n}(x) \leq 2 c^{2} \Lambda+e^{-\alpha x_{N}} H_{h_{n} \chi_{V\left(r^{\prime}\right)}}^{A\left(r^{\prime}, 1\right)}(x) .
$$

We choose $c_{5}=2 c^{2}$ and let $n \rightarrow \infty$. By (2.23) the limit of the latter term on the right hand side of (2.24) is finite and so $H_{v}^{V}$ exists and satisfies

$$
H_{v}^{V}(x) \leq c_{5} \Lambda e^{\alpha x_{N}}+H_{v}^{A\left(r^{\prime}, 1\right)}(x) \quad\left(\left|x^{\prime}\right|=r\right) .
$$

Lemma 2.5. Let $w: \partial^{\infty} U \rightarrow[0,+\infty)$ be a Borel measurable function such that

$$
w(y) \leq \beta_{E^{\prime}}(y) e^{\alpha y_{N}} \quad(y \in \partial U) \quad \text { and } \quad w(\infty)=0 .
$$

Then, there exists a positive constant $c_{6}$, depending on $N, R, r$ and $\nu$, such that

$$
H_{w}^{U}\left(x^{\prime}, x_{N}\right) \leq c_{6} e^{\alpha x_{N}} \Lambda \quad\left(\left|x^{\prime}\right|=r\right) .
$$

Proof. Using (2.2), in view of (2.25) and (2.15), for $\left|x^{\prime}\right|=r$ we have

$$
\begin{aligned}
H_{w}^{U}\left(x^{\prime}, x_{N}\right) & \leq C(N, r) \int_{\partial U} w(y) e^{-\alpha\left|y_{N}-x_{N}\right|} d \sigma(y) \\
& \leq C(N, r) e^{\alpha x_{N}} \int_{\partial U} \beta_{E^{\prime}}(y) d \sigma(y) \leq C(N, R, r, \nu) e^{\alpha x_{N}} \Lambda .
\end{aligned}
$$

We extend $h_{+}$to be 0 outside $U$ and recall that $V$ stands for $A\left(r^{\prime}, \infty\right) \backslash E^{\prime}$. We define inductively a sequence $\left(s_{k}\right)$ as follows

$$
\begin{aligned}
s_{-2} & =s_{-1}=0, \quad s_{0}=h_{+}, \\
s_{2 k+1} & =\left\{\begin{array}{ll}
\bar{H}_{s_{2 k}}^{V} & \text { on } V, \\
s_{2 k} & \text { on } \mathbf{R}^{N} \backslash V,
\end{array} s_{2 k+2}= \begin{cases}\bar{H}_{s_{2 k+1}}^{U}+h_{+} & \text {on } U, \\
s_{2 k+1} & \text { on } \mathbf{R}^{N} \backslash U .\end{cases} \right.
\end{aligned}
$$

We put $s_{k}(\infty)=0$ for all $k$.

Lemma 2.6. There is a positive constant $c_{7}$, depending on $N, R, r, r^{\prime}$ and $\nu$, such that, if $\Lambda \leq c_{7} \lambda$ for some $\lambda \in(0,1)$, then:

(a) $\left(s_{k}\right)$ is an increasing sequence of continuous functions on $\mathbf{R}^{N}$; 
(b) each $s_{k}$ is bounded on $\mathbf{R}^{N-1} \times\left(-\infty, b_{n}\right)$ for each $n \in \mathbf{N}$;

(c) for all $k=0,1, \ldots$ we have

$$
\left(s_{2 k}-s_{2 k-2}\right)(x) \leq \lambda^{k} e^{\alpha x_{N}}, \quad\left|x^{\prime}\right|=r .
$$

Proof. We will use ideas from [18, Lemma 3.1]. Suppose that $\Lambda \leq c_{7} \lambda$, where $c_{7}$ is to be determined later. Assume that $s_{0} \leq s_{1} \leq \ldots \leq s_{2 k}$ on $\mathbf{R}^{N}$ for some $k \geq 0$, that all the functions $s_{k^{\prime}}$ are continuous on $\mathbf{R}^{N}$ for $0 \leq k^{\prime} \leq 2 k$, and that for $0 \leq k^{\prime} \leq k$

$$
\left(s_{2 k^{\prime}}-s_{2 k^{\prime}-2}\right)\left(x^{\prime}, x_{N}\right) \leq \lambda^{k^{\prime}} e^{\alpha x_{N}} \quad\left(\left|x^{\prime}\right|=r\right) .
$$

We also fix $n \in \mathbf{N}$ and assume that $s_{2 k}$ is bounded on $\mathbf{R}^{N-1} \times\left(-\infty, b_{n}\right)$. Once the terms of $\left(s_{k}\right)$ are seen to be finite, it is clear that the upper PWB solutions appearing in their definitions are actually well defined PWB solutions. The induction hypotheses clearly hold for $k=0$. We split the proof of Lemma 2.6 into three steps.

Step 1. We show that $s_{2 k+1}$ is a finite-valued continuous function on $\mathbf{R}^{N}$ which is bounded on $\mathbf{R}^{N-1} \times\left(-\infty, b_{n}\right)$. Harnack's inequalities and (2.26) yield the existence of a constant $c_{8}=c_{8}\left(N, r, r^{\prime}\right)>0$ such that

$$
\left(s_{2 k}-s_{2 k-2}\right)(y) \leq c_{8} \lambda^{k} e^{\alpha y_{N}} \quad\left(\left|y^{\prime}\right|=r^{\prime}\right) .
$$

Now, for $\left|x^{\prime}\right|=r$, by (2.27) and Lemma 2.4 we have

$$
\begin{aligned}
\left(s_{2 k+1}-s_{2 k-1}\right)(x) & \leq \bar{H}_{s_{2 k}-s_{2 k-2}}^{V}(x)=\bar{H}_{\left(s_{2 k}-s_{2 k-2}\right) \chi_{V\left(r^{\prime}\right)}}^{V}(x) \\
& \leq c_{5} c_{8} \lambda^{k} \Lambda e^{\alpha x_{N}}+H_{\left(s_{2 k}-s_{2 k-2}\right) \chi_{V\left(r^{\prime}\right)}(x) .}^{\left(r^{\prime}, 1\right)}(x)
\end{aligned}
$$

Since $s_{2 k}-s_{2 k-1}=0$ on $\partial U$ and $s_{2 k}-s_{2 k-1}=s_{2 k}-s_{2 k-2}$ on $V\left(r^{\prime}\right)$, it follows that $s_{2 k}-s_{2 k-1}$ belongs to the upper class for $H_{\left(s_{2 k}-s_{2 k-2}\right) \chi_{V\left(r^{\prime}\right)}}^{A\left(r^{\prime}, 1\right)}$. Hence

$$
\left(s_{2 k+1}-s_{2 k-1}\right)(x) \leq c_{5} c_{8} \lambda^{k} \Lambda e^{\alpha x_{N}}+\left(s_{2 k}-s_{2 k-1}\right)(x)
$$

and so

$$
\left(s_{2 k+1}-s_{2 k}\right)(x) \leq c_{5} c_{8} \lambda^{k} \Lambda e^{\alpha x_{N}} \quad\left(\left|x^{\prime}\right|=r\right) .
$$

This proves finiteness of $s_{2 k+1}$.

A result of Armitage concerning a strong type of regularity for the PWB solution of the Dirichlet problem (see [3, Theorem 2]) implies that $s_{2 k+1}$ is continuous at points of $\partial V \backslash \bigcup_{n=1}^{\infty}\left(\partial B^{\prime} \times\left\{b_{n}\right\}\right)$. Applying Lemma 2.3 to $v_{j}=H_{\min \left\{s_{2 k}, j\right\}}^{V}$ and $x \in \bigcup_{n=1}^{\infty}\left(\partial B^{\prime} \times\left\{b_{n}\right\}\right)$ we obtain

$$
v_{j}(y) \leq c_{3} v_{j}\left(r x^{\prime}, x_{N}\right) H_{\chi \partial T_{x}}^{T_{x} \backslash E^{\prime}}(y) \quad\left(y \in T_{x} \backslash E^{\prime}\right) .
$$

Letting $j \rightarrow \infty$ we notice that the same inequality holds for $s_{2 k+1}$, and hence the regularity of $x$ for $T_{x} \backslash E^{\prime}$ implies that $s_{2 k+1}$ vanishes at $x$. We conclude that $s_{2 k+1}$ is continuous on $\mathbf{R}^{N}$.

We also have $s_{2 k+1}=H_{s_{2 k+1}}^{V \cap\left[\mathbf{R}^{N-1} \times\left(-\infty, b_{n}\right)\right]}$ on $V \cap\left[\mathbf{R}^{N-1} \times\left(-\infty, b_{n}\right)\right]$. Further, since $s_{2 k+1}$ is continuous on $\overline{B^{\prime}} \times\left\{b_{n}\right\}$, vanishes on $E$ and is bounded on $\left(\mathbf{R}^{N} \backslash\right.$ $V) \cap\left[\mathbf{R}^{N-1} \times\left(-\infty, b_{n}\right)\right]$ in view of the induction hypothesis, we deduce that $s_{2 k+1}$ is bounded above on $\mathbf{R}^{N-1} \times\left(-\infty, b_{n}\right)$.

Step 2. We now prove that $s_{2 k} \leq s_{2 k+1} \leq s_{2 k+2}$ on $\mathbf{R}^{N}$. We note that $s_{2 k}=$ $H_{s_{2 k}}^{A\left(r^{\prime}, 1\right)}$ on $A\left(r^{\prime}, 1\right)$ (for a simple proof see Step 2 in the proof of [18, Lemma 3.1]). 
It follows immediately from the induction hypothesis, that

$$
s_{2 k+1}=H_{s_{2 k}}^{V} \geq H_{s_{2 k-2}}^{V}=s_{2 k-1} \quad \text { on } \quad V .
$$

In particular, this gives $s_{2 k+1} \geq s_{2 k}$ on $\mathbf{R}^{N} \backslash U$. Hence, $s_{2 k+1} \geq s_{2 k}$ on $\partial U \cup \partial V$. Using [5, Theorem 6.3.6], we obtain

$$
s_{2 k+1}=H_{s_{2 k}}^{V}=H_{s_{2 k+1}}^{A\left(r^{\prime}, 1\right)} \geq H_{s_{2 k}}^{A\left(r^{\prime}, 1\right)}=s_{2 k} \quad \text { on } \quad A\left(r^{\prime}, 1\right) .
$$

Therefore, $s_{2 k+1} \geq s_{2 k}$ on $\mathbf{R}^{N}$. We now deduce that

$$
s_{2 k+2}=\bar{H}_{s_{2 k+1}}^{U}+h_{+} \geq H_{s_{2 k-1}}^{U}+h_{+}=s_{2 k}=s_{2 k+1} \quad \text { on } \quad \mathbf{R}^{N} \backslash V .
$$

We finally note that, if $s_{2 k+2}$ belongs to the upper class for $\bar{H}_{s_{2 k+2}}^{A\left(r^{\prime}, 1\right)}$, we obtain

$$
s_{2 k+2} \geq \bar{H}_{s_{2 k+2}}^{A\left(r^{\prime}, 1\right)} \geq H_{s_{2 k+1}}^{A\left(r^{\prime}, 1\right)}=s_{2 k+1} \quad \text { on } A\left(r^{\prime}, 1\right),
$$

and so $s_{2 k+2} \geq s_{2 k+1}$ on $\mathbf{R}^{N}$. To verify that $s_{2 k+2}$ belongs to the upper class for $\bar{H}_{s_{2 k+2}}^{A\left(r^{\prime}, 1\right)}$ it is enough to check that $\liminf _{x \rightarrow y} s_{2 k+2}(x) \geq s_{2 k+2}(y)$ for $y \in \partial U$. This is clear from regularity and the continuity of $s_{2 k+1}$, as if $s_{2 k+2} \not \equiv+\infty$, then for $y \in \partial U$ we have

$$
\liminf _{x \rightarrow y} s_{2 k+2}(x)=\liminf _{x \rightarrow y} H_{s_{2 k+1}}^{U}(x) \geq \liminf _{x \rightarrow y, x \in \partial U} s_{2 k+1}(x)=s_{2 k+1}(y)=s_{2 k+2}(y) .
$$

Step 3. In the final step we will prove that

$$
\left(s_{2 k+2}-s_{2 k}\right)(x) \leq \lambda^{k+1} e^{\alpha x_{N}} \quad\left(\left|x^{\prime}\right|=r\right) .
$$

Then, using [3, Theorem 2], we can conclude that $s_{2 k+2}$ is continuous on $\mathbf{R}^{N}$. Further, $s_{2 k+2}-h_{+}=H_{s_{2 k+1}}^{U}=H_{s_{2 k+2}-h_{+}}^{U \cap\left[\mathbf{R}^{N-1} \times\left(-\infty, b_{n}\right)\right]}$ on $U \cap\left[\mathbf{R}^{N-1} \times\left(-\infty, b_{n}\right)\right]$. By continuity, $s_{2 k+2}$ is bounded on $\overline{B^{\prime}} \times\left\{b_{n}\right\}$. On $\mathbf{R}^{N} \backslash U$ we have $s_{2 k+2}=s_{2 k+1}$, which is bounded on $\left(\mathbf{R}^{N} \backslash U\right) \cap\left[\mathbf{R}^{N-1} \times\left(-\infty, b_{n}\right)\right]$ by Step 1 . Hence $s_{2 k+2}$ is bounded on the whole of $\mathbf{R}^{N-1} \times\left(-\infty, b_{n}\right)$.

To prove the desired inequality (2.29), we first recall that

$$
U_{m}=\left(\mathbf{R}^{N-1} \backslash \overline{B^{\prime}}\right) \times\left(b_{m}, b_{m+1}\right) \quad(m \in \mathbf{N}) .
$$

Noting that

$$
s_{2 k+1}=H_{s_{2 k}}^{V}=H_{s_{2 k+1}}^{U_{m}}=H_{s_{2 k+1} \chi_{\partial B^{\prime} \times\left(b_{m}, b_{m}+1\right)}}^{U_{m}} \quad \text { on } U_{m},
$$

and that, by continuity, $s_{2 k+1}$ is bounded on $\partial B^{\prime} \times\left(b_{m}, b_{m+1}\right)$, we see that $s_{2 k+1}-s_{2 k-1}$ satisfies the hypotheses of Lemma 2.3. Hence, for $x \in \partial U$, we have

$$
\begin{aligned}
\left(s_{2 k+1}-s_{2 k-1}\right)(x) & \leq c_{3} \beta_{E^{\prime}}(x)\left(s_{2 k+1}-s_{2 k-1}\right)\left(r x^{\prime}, x_{N}\right) \\
& =c_{3} \beta_{E^{\prime}}(x)\left[\left(s_{2 k+1}-s_{2 k}\right)\left(r x^{\prime}, x_{N}\right)+\left(s_{2 k}-s_{2 k-1}\right)\left(r x^{\prime}, x_{N}\right)\right] \\
& \leq c_{3} \beta_{E^{\prime}}(x)\left[\left(s_{2 k+1}-s_{2 k}\right)\left(r x^{\prime}, x_{N}\right)+\left(s_{2 k}-s_{2 k-2}\right)\left(r x^{\prime}, x_{N}\right)\right] .
\end{aligned}
$$

It follows from (2.28) and our induction hypothesis that

$$
\left(s_{2 k+1}-s_{2 k-1}\right)(x) \leq c_{3}\left(c_{5} c_{8} \Lambda+1\right) \lambda^{k} e^{\alpha x_{N}} \beta_{E^{\prime}}(x) \quad(x \in \partial U) .
$$

Assuming that $c_{7} \leq 1$ and letting $c_{9}=c_{3}\left(c_{5} c_{8}+1\right)$ we obtain

$$
\left(s_{2 k+1}-s_{2 k-1}\right)(x) \leq c_{9} \lambda^{k} e^{\alpha x_{N}} \beta_{E^{\prime}}(x) \quad(x \in \partial U) .
$$


By Lemma 2.5, for $\left|x^{\prime}\right|=r$, we have

$$
\left(s_{2 k+2}-s_{2 k}\right)(x) \leq \bar{H}_{s_{2 k+1}-s_{2 k-1}}^{U}(x) \leq c_{9} \lambda^{k} c_{6} \Lambda e^{\alpha x_{N}}=c_{6} c_{7} c_{9} \lambda^{k+1} e^{\alpha x_{N}} .
$$

Taking $c_{7}=\min \left\{1,\left(c_{6} c_{9}\right)^{-1}\right\}$ we find that (2.29) holds, and the proof is complete.

\section{Proof of Theorem 1.1}

Proposition 2.1 gives the implication $(a) \Rightarrow(b)$. To prove that $(b) \Rightarrow(a)$ we first observe that taking $J$ large enough when setting $b_{1}=a_{J}$, we can ensure that $\Lambda \leq c_{7} \lambda$ for some $\lambda \in(0,1)$. Let $\Omega^{\prime}=\mathbf{R}^{N} \backslash E^{\prime}$ and $u^{\prime}=\lim _{k \rightarrow \infty} s_{k}$. By Lemma 2.6, for $\left|x^{\prime}\right|=r$ we obtain

$$
s_{2 k}(x)=\sum_{j=0}^{k}\left(s_{2 j}-s_{2 j-2}\right)(x) \leq \sum_{j=0}^{k} \lambda^{j} e^{\alpha x_{N}} \leq \frac{1}{1-\lambda} e^{\alpha x_{N}} .
$$

Hence $u^{\prime} \not \equiv+\infty$. As a limit of an increasing sequence $\left(s_{2 k}\right)$ of harmonic functions on $U$, the function $u^{\prime}$ is harmonic on $U$. Since $u^{\prime}$ is the limit of an increasing sequence $\left(s_{2 k+1}\right)$ of harmonic functions on $V$, it is also harmonic on $V$. Hence $u^{\prime}$ is harmonic in $\Omega^{\prime}$. It follows from the monotonicity of $\left(s_{k}\right)$ that $u^{\prime} \geq h_{+}$on $U$.

For $x \in E^{\prime}$ we have $u^{\prime}(x)=0$. By the monotone convergence theorem applied to the equation $s_{2 k+1}=H_{s_{2 k}}^{V}$ we obtain $u^{\prime}=H_{u^{\prime}}^{V}$ on $V$. We can follow the reasoning from the second last paragraph of Step 1 in the proof of Lemma 2.6 to see that $u^{\prime}$ vanishes continuously on $E^{\prime}$.

We next prove that $u^{\prime}$ is minimal on $\Omega^{\prime}$ using an argument from [18, Theorem 1.1]. As a consequence of the monotone convergence theorem we find that

$$
u^{\prime}(x)=H_{u^{\prime}}^{U}(x)+h_{+}(x) \quad(x \in U) .
$$

Let $\Delta_{1}$ denote the minimal Martin boundary of $\Omega^{\prime}$ and let $M$ be the Martin kernel of $\Omega^{\prime}$ relative to the origin. By the Martin representation theorem (see [5, Theorem 8.4.1]) we have

$$
u^{\prime}(x)=\int_{\Delta_{1}} M(x, z) d \nu_{u^{\prime}}(z) \quad\left(x \in \Omega^{\prime}\right)
$$

where $\nu_{u^{\prime}}$ is uniquely determined by $u^{\prime}$.

We define $T=\left\{z \in \Delta_{1}: \Omega^{\prime} \backslash U\right.$ is minimally thin at $\left.z\right\}$ so that

$$
R_{M(\cdot, z)}^{\Omega^{\prime} \backslash U}=M(\cdot, z) \quad\left(z \in \Delta_{1} \backslash T\right) .
$$

Changing the order of integration, and using (3.1)-(3.3) and [5, Theorem 6.9.1], we obtain

$$
\begin{aligned}
h_{+}(x) & =\int_{\Delta_{1}}\left(M(x, z)-\int_{\partial U} M(y, z) d \mu_{x}^{U}(y)\right) d \nu_{u^{\prime}}(z) \\
& =\int_{\Delta_{1}}\left(M(x, z)-R_{M(\cdot, z)}^{\Omega^{\prime} \backslash U}(x)\right) d \nu_{u^{\prime}}(z) \\
& =\int_{T}\left(M(x, z)-R_{M(\cdot, z)}^{\Omega^{\prime} \backslash U}(x)\right) d \nu_{u^{\prime}}(z) \quad(x \in U) .
\end{aligned}
$$


We now claim that $\left.\nu_{u^{\prime}}\right|_{T}$ is concentrated at a single point. For the sake of contradiction suppose that there are two distinct points $y_{1}, y_{2} \in \Delta_{1} \cap \operatorname{supp}\left(\left.\nu_{u^{\prime}}\right|_{T}\right)$ and let $N_{1}, N_{2}$ be disjoint neigbourhoods of $y_{1}$ and $y_{2}$ respectively. We define

$$
h_{j}(x)=\int_{N_{j} \cap T}\left(M(x, y)-R_{M(\cdot, y)}^{\Omega^{\prime} \backslash U}(x)\right) d \nu_{u^{\prime}}(y) \quad\left(x \in \Omega^{\prime}, j=1,2\right),
$$

and note that $h_{j} \leq h_{+}$on $U$. Minimality of $h_{+}$on $U$ implies that

$$
h_{j} / h_{j}(0)=h_{+} \quad \text { on } U \quad(j=1,2) .
$$

We now define

$$
v_{j}(x)=\int_{N_{j} \cap T} M(x, y) d \nu_{u^{\prime}}(y) \quad\left(x \in \Omega^{\prime}, j=1,2\right) .
$$

Then $h_{j} \leq v_{j} \leq u^{\prime}$ on $\Omega^{\prime}$, and by (3.4), $v_{j} / h_{j}(0) \geq h_{+}$on $\Omega^{\prime}(j=1,2)$. In view of the definition of $s_{k}$ we have $v_{j} / h_{j}(0) \geq s_{k}$ on $\Omega^{\prime}$ for all $k \in \mathbf{N}$ and so $v_{j} / h_{j}(0) \geq u^{\prime}$ on $\Omega^{\prime}(j=1,2)$. It follows that $h_{1}(0) v_{2} \leq v_{1}$ on $\Omega^{\prime}$. This implies that $\left.\nu_{u^{\prime}}\right|_{T \cap N_{1}}$ is minorized by a multiple of $\left.\nu_{u^{\prime}}\right|_{T \cap N_{2}}$, which contradicts the fact that $N_{1} \cap N_{2}=\emptyset$. Hence $\left.\nu_{u^{\prime}}\right|_{T}=c \delta_{t^{\prime}}$ for some $t^{\prime} \in T$ and $c>0$. Furthermore, the minimal harmonic function $v=c M\left(\cdot, t^{\prime}\right)$ on $\Omega^{\prime}$ satisfies $u^{\prime} \geq v$ on $\Omega^{\prime}$ and $v \geq h_{+}$on $U$. We observe that $v \geq s_{k}$ on $\Omega^{\prime}$ for all $k \in \mathbf{N}$, and so $v \geq u^{\prime}$. Hence $v \equiv u^{\prime}$ and we conclude that $u^{\prime}$ is minimal on $\Omega^{\prime}$.

Let $\Omega^{\prime \prime}=\mathbf{R}^{N} \backslash E^{\prime \prime}$. We define $g=H_{\chi_{\Omega^{\prime \prime} \backslash \Omega^{\prime}}^{\Omega^{\prime}}}$ and $g=\chi_{\Omega^{\prime \prime} \backslash \Omega^{\prime}}$ on $\partial^{\infty} \Omega^{\prime}$. By [5, Theorem 6.9.1] we have $g=R_{1}^{\Omega^{\prime \prime} \backslash \Omega^{\prime}}$ on $\Omega^{\prime \prime}$ (reductions with respect to nonnegative superharmonic functions on $\Omega^{\prime \prime}$ ). Since $\Omega^{\prime \prime} \backslash \Omega^{\prime}$ is non-thin at each constituent point, it follows from [5, Theorem 7.3.1(i)] that $R_{1}^{\Omega^{\prime \prime} \backslash \Omega^{\prime}}=\widehat{R}_{1}^{\Omega^{\prime \prime} \backslash \Omega^{\prime}}$ on $\Omega^{\prime \prime}$ and so $g$ is superharmonic there. Let $h$ be a non-negative harmonic minorant of $g$ on $\Omega^{\prime \prime}$. Then $h$ is bounded on $\Omega^{\prime \prime}$ and vanishes quasi-everywhere on $\partial \Omega^{\prime \prime}$. Since a polar subset of $\partial \Omega^{\prime \prime}$ and $\{\infty\}$ are both negligible for $\Omega^{\prime \prime}$ (see [5, Theorems 6.5.5 and 7.6.5]), we deduce that $h \equiv 0$. Hence $g$ is a potential on $\Omega^{\prime \prime}$.

Let $W=\left[\mathbf{R}^{N-1} \times\left(-\infty, b_{n}\right)\right] \cap \Omega^{\prime}$ for some $n>1$. Since $1-g$ is positive and continuous on $\overline{B^{\prime}} \times\left\{b_{n}\right\}$, it follows that $1-g$ is bounded below by a positive constant on this set while $u^{\prime}$ is bounded from above there. Hence there exists a positive constant $c$ such that $c(1-g) \geq u^{\prime}$ on $\overline{B^{\prime}} \times\left\{b_{n}\right\}$, and thus on $\partial W$. By Lemma 2.6(b) each $s_{k}$ is bounded on $W$ and so it belongs to the lower class for $H_{s_{k}}^{W}$. These facts combined with monotonicity of $\left(s_{k}\right)$ lead to the observation that

$$
s_{k} \leq H_{s_{k}}^{W} \leq H_{u^{\prime}}^{W} \leq c H_{1-g}^{W}=c(1-g) \text { on } W .
$$

Therefore, $u^{\prime} \leq c(1-g)$ on $W$. Since $c(1-g)-u^{\prime}$ is a non-negative harmonic function on $W$ which vanishes on $\Omega^{\prime \prime} \backslash \Omega^{\prime}$, we conclude that $c(1-g)-u^{\prime}$ is subharmonic on $\Omega^{\prime \prime}$, so that $u^{\prime}+c g$ is superharmonic on $\Omega^{\prime \prime}$.

By the Riesz decomposition,

$$
u^{\prime}+c g=u^{\prime \prime}+G_{\Omega^{\prime \prime}} \mu \text { on } \Omega^{\prime \prime}
$$

where $u^{\prime \prime}$ is the greatest harmonic minorant of $u^{\prime}+c g$ on $\Omega^{\prime \prime}$ and $G_{\Omega^{\prime \prime}} \mu$ is the Green potential of the Riesz measure $\mu$ associated with $u^{\prime}+c g$. Hence $u^{\prime \prime}$ vanishes on $E^{\prime \prime} \backslash\left(\partial B^{\prime} \times\left\{b_{1}\right\}\right)$ and for each $n \in \mathbf{N}$ it is bounded on $\mathbf{R}^{N-1} \times\left(-\infty, b_{n}\right)$. It follows from a removable singularity result (see [5, Theorem 5.2.1]) that $u^{\prime \prime}$ extends to a subharmonic function on $\mathbf{R}^{N}$. This together with the non-thinness of $E^{\prime \prime}$ at points 
of $\partial B^{\prime} \times\left\{b_{1}\right\}$ implies that $u^{\prime \prime}$ vanishes also on $\partial B^{\prime} \times\left\{b_{1}\right\}$. Since $h_{+}$is a subharmonic minorant of $u^{\prime}+c g$ on $\Omega^{\prime \prime}$, we deduce that $h_{+} \leq u^{\prime \prime}$ on $\Omega^{\prime \prime}$.

It remains to show that $u^{\prime \prime}$ is minimal. Let $h$ be a positive harmonic minorant of $u^{\prime \prime}$ on $\Omega^{\prime \prime}$. We notice that $h$ is bounded on $\Omega^{\prime \prime} \backslash \Omega^{\prime}$ and vanishes on $\partial \Omega^{\prime \prime}$. Hence the greatest harmonic minorant of $R_{h}^{\Omega^{\prime \prime} \backslash \Omega^{\prime}}$ on $\Omega^{\prime \prime}$ is bounded and vanishes on $\partial \Omega^{\prime \prime}$, and we see that $R_{h}^{\Omega^{\prime \prime} \backslash \Omega^{\prime}}$ is a potential on $\Omega^{\prime \prime}$. Since the upper-bounded harmonic function $h-R_{h}^{\Omega^{\prime \prime} \backslash \Omega^{\prime}}-u^{\prime}$ on $\Omega^{\prime}$ satisfies

$$
\limsup _{x \rightarrow y}\left(h-R_{h}^{\Omega^{\prime \prime} \backslash \Omega^{\prime}}-u^{\prime}\right)(x) \leq 0 \quad \text { for } y \in \partial \Omega^{\prime},
$$

and $\{\infty\}$ has zero harmonic measure for $\Omega^{\prime}$, it follows that

$$
h-R_{h}^{\Omega^{\prime \prime} \backslash \Omega^{\prime}}-u^{\prime} \leq 0 \quad \text { on } \Omega^{\prime} .
$$

Now, since $h-R_{h}^{\Omega^{\prime \prime} \backslash \Omega^{\prime}}$ is a positive harmonic minorant of the minimal function $u^{\prime}$ on $\Omega^{\prime}$, we conclude that $h-R_{h}^{\Omega^{\prime \prime} \backslash \Omega^{\prime}}=a u^{\prime}$ for some $a \in(0,1]$. Substituting this into (3.5) we obtain

$$
h+a c g=a u^{\prime \prime}+a G_{\Omega^{\prime \prime}} \mu+R_{h}^{\Omega^{\prime \prime} \backslash \Omega^{\prime}} \text { on } \Omega^{\prime \prime} .
$$

Taking the greatest harmonic minorant in $\Omega^{\prime \prime}$ of both sides we get $h=a u^{\prime \prime}$, which means that $u^{\prime \prime}$ is minimal.

Let $u=u^{\prime \prime}-H_{u^{\prime \prime}}^{\Omega}$. Since $u^{\prime \prime}-h_{+} \geq 0$ is superharmonic on $\Omega^{\prime \prime}$ and equals $u^{\prime \prime}$ on $\Omega^{\prime \prime} \backslash \Omega$, we have

$$
u=u^{\prime \prime}-R_{u^{\prime \prime}}^{\Omega^{\prime \prime} \backslash \Omega}=u^{\prime \prime}-R_{u^{\prime \prime}-h_{+}}^{\Omega^{\prime \prime}} \geq h_{+} .
$$

Since the points of $\partial \Omega$ are regular for $\Omega$ and $u^{\prime \prime}$ is continuous, it follows that $u$ vanishes on $\partial \Omega$. Further, [5, Theorem 9.5.5] shows that $u$ is minimal.

Remark. The proof of the implication $(a) \Rightarrow(b)$ in Theorem 1.1 does not rely on condition (1.1). It is in the proof of the converse that our methods rely on such a condition. However, it is enough to assume merely that $\Omega$ is contained in a comb-like domain $\Omega_{0}$ for which (1.1) holds. To see this, suppose that (b) holds. Theorem 1.1 applied to $\Omega_{0}$ yields the existence of a minimal harmonic function $u_{0}$ on $\Omega_{0}$ which vanishes on $\partial \Omega_{0}$ and satisfies $u_{0} \geq h_{+}$. Let $u=u_{0}-H_{u_{0}}^{\Omega}$ on $\Omega$. The argument from the previous paragraph shows that $u$ is as stated in $(a)$.

\section{References}

[1] Ancona, A.: Sur la frontière de Martin des domaines de Denjoy. - Ann. Acad. Sci. Fenn. Ser. A I Math. 15:2, 1990, 259-271.

[2] Andrievskit, V. V.: Positive harmonic functions on Denjoy domains in the complex plane. J. Anal. Math. 104, 2008, 83-124.

[3] Armitage, D. H.: A strong type of regularity for the PWB solution of the Dirichlet problem. - Proc. Amer. Math. Soc. 61:2, 1976, 285-289.

[4] Armitage, D. H., and T. B. Fugard: Subharmonic functions in strips. - J. Math. Anal. Appl. 89:1, 1982, 1-27.

[5] Armitage, D. H., and S. J. Gardiner: Classical potential theory. - Springer Monogr. Math., Springer-Verlag, London, 2001.

[6] Benedicks, M.: Positive harmonic functions vanishing on the boundary of certain domains in $\mathbf{R}^{N}$. - Ark. Mat. 18:1, 1980, 53-72. 
[7] Burdzy, K.: Brownian excursions and minimal thinness. Part III: Application to the angular derivative problem. - Math. Z. 192:1, 1986, 89-107.

[8] Carleson, L., and V. Totik: Hölder continuity of Green's functions. - Acta Sci. Math. (Szeged) 70:3-4, 2004, 557-608.

[9] Carroll, T.: A classical proof of Burdzy's theorem on the angular derivative. - J. London Math. Soc. 38:3, 1988, 423-441.

[10] Carroll, T., and S. J. Gardiner: Lipschitz continuity of the Green function in Denjoy domains. - Ark. Mat. 46:2, 2008, 271-283.

[11] Chevaluier, N.: Frontière de Martin d'un domaine de $\mathbf{R}^{N}$ dont le bord est inclus dans une hypersurface lipschitzienne. - Ark. Mat. 27:1, 1989, 29-48.

[12] Cranston, M. C., and T. S. Salisbury: Martin boundaries of sectorial domains. - Ark. Mat. $31: 1,1993,27-49$.

[13] Domar, Y.: On the existence of a largest subharmonic minorant of a given function. - Ark. Mat. 3, 1957, 429-440.

[14] Gardiner, S. J.: Minimal harmonic functions on Denjoy domains. - Proc. Amer. Math. Soc. 107:4, 1989, 963-970.

[15] Gardiner, S. J.: The Martin boundary of NTA strips. - Bull. London Math. Soc. 22:2, 1990, $163-166$.

[16] Gardiner, S. J.: A short proof of Burdzy's theorem on the angular derivative. - Bull. London Math. Soc. 23:6, 1991, 575-579.

[17] Gardiner, S. J., and W. Hansen: The Riesz decomposition of finely superharmonic functions. - Adv. Math. 214:1, 2007, 417-436.

[18] Ghergu, M., and J. Pres: Positive harmonic functions that vanish on a subset of a cylindrical surface. - Potential Anal. 31:2, 2009, 147-181.

[19] Henrot, A.: Extremum problems for eigenvalues of elliptic operators. - Birkhäuser Verlag, Basel, 2006.

[20] Jenkins, J. A.: On comb domains. - Proc. Amer. Math. Soc. 124:1, 1996, 187-191.

[21] Lömker, A.: Martin boundaries of quasi-sectorial domains. - Potential Anal. 13:1, 2000, 1167.

[22] Rodin, B., and S. E. Warschawski: Extremal length and univalent functions. The angular derivative. - Math. Z. 153:1, 1977, 1-17.

[23] Rodin, B., and S. E. Warschawski: Angular derivative conditions for comb domains. - In: Topics in complex analysis (Fairfield, Conn., 1983), Contemp. Math. 38, 1985, 61-68.

[24] Segawa, S.: Martin boundaries of Denjoy domains. - Proc. Amer. Math. Soc. 103:1, 1988, $177-183$.

[25] Segawa, S.: Martin boundaries of Denjoy domains and quasiconformal mappings. - J. Math. Kyoto Univ. 30:2, 1990, 297-316. 